\section{Fulminant early-onset neonatal sepsis due to Streptococcus pneumoniae: Case report and review of the literature}

\author{
Carlos Aldana-Valenzuela, ${ }^{1}$ \\ Andrea Marina Rodriguez-López, ${ }^{2}$ \\ Erika Guillén- Blancas ${ }^{1}$ \\ 1Department of Neonatology and \\ 2Department of Epidemiology, Mothers \\ and Children Hospital of León, \\ Guanajuato, México
}

\begin{abstract}
Streptococcus pneumoniae is a rare cause of neonatal sepsis, and it is associated with significant morbidity and a very high fatality rate. The infection is usually acquired intrapartum, from the colonization of the maternal genital tract. Most affected neonates have an early-onset presentation of symptoms, usually within the first 48 hours after birth, which is similar to other causes of neonatal sepsis such as Streptococcus Agalactiae or Escherichia Coli. However, the virulence seems to be higher for Streptococcus pneumoniae, which has in addition a higher infant invasion/maternal colonization ratio than Streptococcus Agalactiae. Pneumococcal vaccination has not resulted in a significant decline of neonatal cases. Many cases included ours, involved serotypes not present in the vaccine. Other strategies to protect these infants are necessary. We describe a late preterm infant with a fatal, early-onset sepsis caused by Streptococcus Pneumoniae serotype 28 A. Maternal vaginal culture grew the same bacteria.
\end{abstract}

\section{Introduction}

Streptococcus Pneumoniae neonatal sepsis (SPNS) is a rare cause of neonatal sepsis, and is associated with high morbidity and mortality.1,2 SPNS usually has an early-onset presentation, and rarely manifests as a late presentation. It is clinically indistinguishable, from other causes of early-onset neonatal sepsis and frequently has a very aggressive clinical course. Strategies to prevent Streptococcus Pneumoniae are evolving, so it is important to acquire more knowledge about this frequently lethal disease, which could help develop effective preventive strategies. We describe a case of a late preterm infant with early-onset Streptococcus Pneumoniae sepsis which proved to be lethal.

\section{Case Report}

A 19-year-old woman, gravida 2, delivered vaginally, a $1950 \mathrm{~g}$ girl at 34 weeks gestation. She had 6 prenatal consultations and the pregnancy was uneventful. No intrapartum antibiotics were given. Membranes ruptured spontaneously six hours before delivery, and there was no maternal fever. The infant was born vigorous with Apgar scores of 8 at one minute and 9 at five minutes. Mild tachypnea was noted and the infant was admitted in the Intermediate Care Nursery for observation. She was pink, active and with good peripheral perfusion and initially she was treated with blow-by oxygen, achieving oxygen saturations $>90 \%$. The tachypnea was transient and lasted only three hours. Enteral feedings were withheld and a peripheral venous catheter was inserted. At 18 hours of age the infant had severe apnea, mottled skin and delayed capillary refill time. She was quickly intubated, and placed on mechanical ventilation. Umbilical artery and umbilical vein catheters were placed. Ampicillin and gentamicin were begun after a blood culture was obtained. Lumbar puncture was not done due to the critical condition of the infant. A chest radiograph showed a reticulogranular pattern in both lung fields, which was suggestive of pneumonia. The clinical course was fulminant, with respiratory failure, severe metabolic acidosis and profound arterial hypotension unresponsive to aggressive fluid resuscitation, and high doses of dopamine and dobutamine. A cell blood count showed leukopenia $\left(2,100 \times \mathrm{mm}^{3}\right)$, neutropenia $(1,400 \times$ $\left.\mathrm{mm}^{3}\right)$ a normal platelet count of $265,000 \times$ $\mathrm{mm}^{3}$ and normal C- reactive protein (4 $\mathrm{mg} / \mathrm{dL}$ ). The infant was pronounced dead at 23 hours of postnatal age. The blood culture grew penicillin-sensitive Streptococcus Pneumoniae 28 A serotype. Mother's vaginal swab was also positive for the same bacteria and serotype.

\section{Discussion and Conclusions}

Neonatal Streptococcus Pneumoniae sepsis is uncommon. It has an estimated frequency that fluctuates between $1-11 \%, 3$ and carries a high mortality that can reach up to $60 \%{ }^{4}$ In contrast to earlier data, ${ }^{4}$ more recent reports have shown that most cases occurred during the first week of life, predominantly within the first 48 hours. ${ }^{5}$

Despite the fact that, SPNS is rare, it seems, as shown by recent data, 6 that the epidemiology of SPNS has changed, since the introduction of 7 (PCV-7) or 13-valent pneumococcal conjugate vaccine (PCV 13).
Correspondence: Carlos Aldana-Valenzuela Departamento de Neonatología, Hospital Materno-Infantil de León, Avenida de la Juventud 116, colonia Jol Gua Ber, 37410, León, Guanajuato, México.

Tel: +52.477.135.6389

E-mail: caldnfp@prodigy.net.mx

Key words: neonates, sepsis, streptococcus pneumoniae.

Acknowledgments: We would like to thank, the staff of the INDRE (Institute of diagnosis and epidemiologic reference) Mexico City, for their identification of pneumococcal serotypes.

Contributions: the authors contributed equally

Conflict of interest: the authors declare no potential conflict of interest.

Funding: none.

Received for publication: 27 November 2018.

Revision received: 6 March 2019.

Accepted for publication: 6 March 2019.

This work is licensed under a Creative Commons Attribution NonCommercial 4.0 License (CC BY-NC 4.0).

(C) Copyright C. Aldana-Valenzuela et al., 2019 Licensee PAGEPress, Italy

Pediatric Reports 2019; 11:7953

doi:10.4081/pr.2019.7953

Olarte et al., ${ }^{7}$ compared the clinical and microbiologic features of affected infants before and after the introduction of this vaccine and observed a decline in the number of cases of approximately 30\%, among infants 0-60 days. They also estimate that $60 \%$ of cases were caused by PCV 13 serotypes, so it is possible that vaccination may provide some protection by passive immunity to neonates born to young mothers immunized before 6 years of age. PCV 13 is included in the Mexican immunization program for children, however, many reported cases are caused by serotypes not included in this vaccine. The 28 A serotype reported in our case is not present in the PCV 13, nor in the 23-valent polysaccharide vaccine 23 ( $\mathrm{PCV} 23$ ) which is used in Mexico for people older than 65 years old, or younger but with some high-risk condition. Pneumococcal vaccine given at birth may be beneficial to prevent late-onset sepsis, although it may not prevent most earlyonset SPNS. 8 Vaccination in pregnancy may be a more promising strategy, ${ }^{9,10}$ although, so far there is insufficient evidence to support this intervention. Further trials are needed. The vaccine type as well as the tim- 
ing of vaccination that could maximize maternal immunogenicity and antibody transfer to the fetus should be addressed by these studies. ${ }^{11}$

Early onset infections can be acquired through a colonized birth canal or rarely, by hematogenous transplacental transmission, secondary to maternal bacteremia. ${ }^{12}$ In our case the mother's genital tract was colonized, so most likely, the infant acquired the infection, through the birth canal. Since Streptococcus Pneumoniae is not normally present in the vagina, colonization of the lower genital tract can occur through orogenital contact with a nasopharyngeal carrier or with a person suffering from acute upper respiratory tract pneumococcal infection. Recognized sepsis risk factors, such as prematurity, prolonged rupture of membranes and clinical chorioamnionitis are not significantly associated with SPNS.13 Despite the rarity of vaginal carriage of Streptococcus Pneumoniae, Gomez et al., 13 noted that in colonized mothers, all infants developed sepsis. This suggests that there is a much higher rate of infection and higher fatality rates from colonized mothers, when compared with Streptococcus Agalactiae. 13,14 Due to this risk, isolation of Streptococcus Pneumoniae from maternal genital tract should be considered pathologic and treated accordingly.

Clinical presentation varies, depending on the postnatal age of the infant. Earlyonset infection closely resembles sepsis caused by other bacteria such as Escherichia coli and Streptococcus Agalactie. Pneumonia, leukopenia and shock, are common manifestations. Meningitis, which is usually associated with late-onset infection, for unknown reasons is more frequent in early onset NSPS as observed by Olarte et al. ${ }^{7}$ Since lumbar puncture was not done in our patient, we do not know if this complication was present. It is recognized, that early onset sepsis has a worse prognosis than late presentation. ${ }^{15}$

In conclusion, strategies to prevent early- onset NSPS are evolving. Some interventions we think are mandatory, these include treatment for all pregnant women with a positive vaginal culture to Streptococcus Pneumoniae, and clinicians' increased awareness of SPNS and prompt aggressive antibiotic treatment of affected neonates. Further surveillance studies, assessment of risk factors for SPNS, as well as evaluation of other strategies such as vaccination during pregnancy, aiming to protect these infants are needed.

\section{References}

1. Nallusamy R. Fatal early-onset neonatal sepsis due to Streptococcus pneumoniae. Med J Malaysia 1998;53:442-5.

2. Primhak RA, Tanner RA, Spencer RC. Pneumococcal infection in the newborn. Arch Dis Child 1993;69:317-8.

3. Fernandez Rodriguez B, Rubio Mascaraque L, Ruiz Fraile L, et al. streptococcus pneumoniae: the forgotten microorganism in neonatal sepsis. Fetal Pediatr Pathol 2015;34:202-5.

4. Hoffman JA, Mason EO, Schultze GE, et al. Streptococcus pneumoniae infections in the neonate. Pediatrics 2003;112:1095-102.

5. Sallam A, Paes B. Streptococcus pneumoniae: an old bug with significant maternal-newborn implications. Am J Perinatol 2004;21:491-5.

6. Ladhani SN, Andrews NJ, Waight P, et al. Impact of the 7-valent pneumococcal conjugate vaccine on invasive pneumococcal disease in infants younger than 90 days in England and Wales. Clin Infect Dis 2013;56:633-40.

7. Olarte L, Barson WJ, Bradley JS, et al. invasive pneumococcal disease in infants aged 0-60 days in the United States in the 13-valent pneumococcal conjugate era. J Pediatric Infect Dis Soc 2018;17:249-52.

8. Scott JA, Ojal J, Ashton L, et al.
Pneumococcal conjugate vaccine given shortly after birth stimulates effective antibody concentrations and primes immunological memory for sustained infant protection. Clin Infec Dis 2011;53:663-70.

9. Faust K, Demmert M, Bendiks M, et al. Intrapartum colonization with Streptococcus Pneumoniae, early-onset sepsis and deficient specific neonatal immune responses. Arch Gynecol Obstet 2012;285:599-604.

10. Jarovsky D, Marchetti IV, Da Silva Mori MA, et al. Early-onset neonatal pneumococcal sepsis: A fatal case report and brief literature review. Pediatr Infect Dis J 2018;37:e111-2.

11. Chaithongwongwatthana S, Yamasmit W, Limpongsanurak $\mathrm{S}$, et al. Pneumococcal vaccination during pregnancy for preventing infant infection. Cochrane Database Syst Rev 2015;1:CD004903.

12. Malhotra A, Hunt RW, Doherty RR. Streptococcus Pneumoniae sepsis in the newborn. J Paediatr Child Health 2012;48:E79-83.

13. Gomez M, Alter S, Kumar ML, et al. Neonatal Streptococcus Pneumoniae infection: Case reports and review of the literature. Pediatr Infect Dis J 1999;18:1014-8.

14. McAdams RM, Garza-Cox S, Yoder BA. Early-onset neonatal pneumococcal sepsis syndrome. Pediatr Crit Care Med 2005;6:595-7.

15. Fothy JF, Vetter S, Iñigo A. Early-onset Streptococcus pneumoniae neonatal sepsis and meningitis in the 13-valent vaccine era. Pediatr Infect Dis J 2013;32:1299-300. 